\title{
Indium-111-labeled leukocytes in the detection of abscesses
}

\author{
EUGENE W. TRYCIECKY, D.O. \\ Owosso, Michigan
}

\section{The experience of an} osteopathic community hospital with indium-111-labeled leukocytes (In-111WBC) for abscess detection is reported. Thirty-two scans were performed in 28 patients with suspected abscesses. Nine true-positive scans were confirmed at surgery, and 16 scans were categorized correctly as true-negatives. One scan was a false-positive, and 2 were falsenegative. The sensitivity of In-111-WBC scanning in this series was 82 percent and the specificity was 94 percent; accuracy was 89 percent. False-negative scans are most likely to occur with septic foci of more than 2 weeks' duration. Twenty-two percent of all scans showed areas of inflammation unrelated to abscesses. In-111-WBC scanning has proved to be a valuable, noninvasive tool in the diagnosis of abscesses, especially acute processes.

The identification of an abscess is a difficult clinical problem. If left untreated, occult abscesses may lead to the patient's death. Traditional imaging modalities for diagnosing abscesses include computed tomography (CT), ultrasonography, and gallium-67 (Ga-67) scintigraphy. Although it is still a relatively new modality, the efficacy of indium-111-labeled leukocyte (In-111-WBC) scintigraphy for abscess detection is well established. ${ }^{1-8}$

This paper reports the experience of an osteopathic community hospital with the use of In-111-WBC scintigraphy in abscess detection.

\section{Materials and methods}

From June 1984 to April 1985, 37 In-111-WBC scintigraphic scans were obtained from 33 patients with suspected abscesses. Each patient's chart was reviewed retrospectively. Five cases were excluded from the final tabulation because of insufficient documentation as to the patient's true condition (that is, presence or absence of an abscess). Therefore, this report is based on the results of 32 scans from 28 patients. The study included 17 females and 11 males, who ranged in age from 15 to 82 years.

At the time of this study, indium-111 oxine was an investigational new drug and approval for its use was obtained from the Food and Drug Administration. (On December 23, 1985, this agent received approval from the FDA for routine clinical use.) Each patient gave informed written consent prior to the procedure. After $40-50 \mathrm{ml}$. of venous blood were drawn into a heparinized syringe, the leukocytes were prepared and labeled with indium-111 oxine by a commercial radiopharmacy, which used the technique described by Beightol and Baker. ${ }^{9} \mathrm{~A}$ total of $300-800 \mu \mathrm{C}$. of In-111-WBCs were injected intravenously into each patient. The total time that elapsed between obtaining the blood to injection of the autologous labeled leukocytes averaged about 3 hours.

Using a Siemens LFOV gamma camera with a medium-energy collimator, imaging was performed 18 to 24 hours after injection. Two 20 -percent windows were set to detect the gamma photon peaks of indium-111 (173 and $247 \mathrm{kev}$ ).

A normal scan demonstrated labeled leukocyte uptake in the spleen, liver, and bone marrow, with uptake being greatest in the spleen and least in the marrow. A scan was considered positive for an abscess if there was an area of abnormal uptake that was equal to or greater in intensity than the uptake in the liver. A scan was considered positive for inflammation, not abscess, if there was an area of abnormal uptake less intense than the liver uptake. 
Representative cases are presented for each category.

\section{Results and case reports}

Table 1 presents the number of scans with each finding (true-negative, true-positive, false-positive, false-negative, or other [inflammation]), as well as the final diagnosis for each category.

\section{True-negative scans}

There were 16 true-negative scans. One negative scan for abscess was confirmed by surgery, 1 by laparoscopy, and 1 by needle biopsy. The remaining 13 scans were confirmed by subsequent clinical correlation. Five patients with normal scans were considered as true-negatives because they became afebrile without antibiotics. Eight patients with normal scans were treated with antibiotics; however, they showed no evidence on other tests to suggest an abscess. Sources for their fevers were documented clinically, and they responded to appropriate therapy and were discharged. They also were considered to be true-negatives.

Case 1. An 18-year-old woman presented with fever and a left adnexal mass on bimanual examination. Culdocentesis was performed, and turbid yellow fluid was obtained. A tubo-ovarian abscess was suspected. An In-111-WBC scan obtained the following
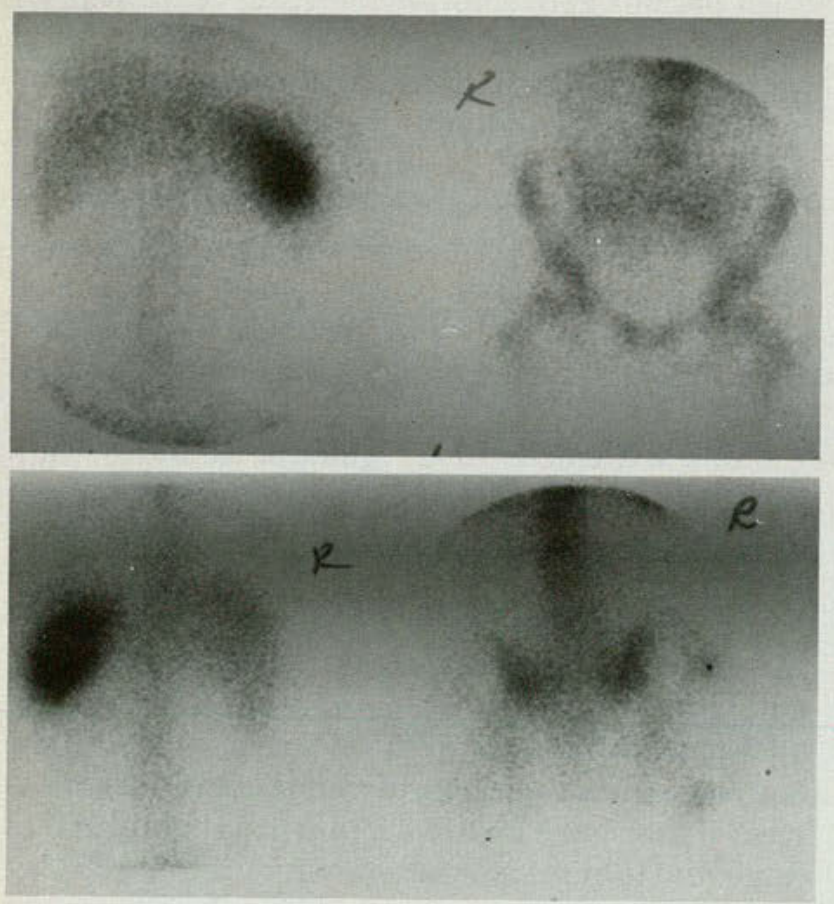

Figs. 1A and 1B. Case 1. [A], Anterior abdominal and pelvic images show normal uptake of In-111-WBCs in the spleen, liver, and bone marrow. Uptake is greatest in the spleen and least in the marrow. No areas of abnormal uptake are present. [B], Normal posterior abdominal and pelvic images. day was normal (Figs. 1A and 1B). One day later, laparoscopy was performed and revealed pelvic inflammatory disease and a left hydrosalpinx. There was no evidence of an abscess. Cultures of the culdocentesis fluid and of fluid obtained at laparoscopy failed to grow organisms.

\section{True-positive scans}

Nine scans were positive for abscess; all of these cases were verified by surgery.

Case 2. A 36-year-old male had a history of Crohn's disease, which had been diagnosed 3 years earlier. The patient had had intermittent fever and chills for about 2 months, and there was recent onset of low-back pain and inability to flex the left leg. A plain film of the abdomen revealed gas bubbles in the left lower quadrant, which were suspected to be extraluminal (Fig. 2A). An In-111-WBC abdominal scan demonstrated abnormal uptake in the left lower quadrant that indicated an abscess (Fig. 2B). This finding was corroborated by a CT scan of the abdomen (Fig. 2C). Surgical exploration disclosed severe Crohn's disease of the sigmoid colon and a left psoas abscess secondary to sigmoid colon perforation.

Case 3. A 42-year-old man developed left lower quadrant pain and fever 14 days after sigmoid colon resection for a perforated diverticulum. An In-111WBC scan performed 4 days later showed a lobulated area of abnormal uptake in the left lower quadrant consistent with abscess, as well as less intense uptake in the descending colon indicating inflammation (Fig. 3A). A barium enema was performed the next day and demonstrated two areas of extravasation of contrast medium from the sigmoid colon, with accumulation of the barium in a large cavity (Fig. 3B). Subsequent surgery revealed an intraperitoneal left lower quadrant abscess, as well as a left rectus muscle abscess, with a fistula between this abscess and the area of previous anastomosis.

\section{False-positive scan}

There was 1 scan positive for abscess, but no abscess was found at subsequent surgery.

Case 4. A 69-year-old man went into septic shock on the night following a transrectal prostate biopsy. The patient's vital signs were stabilized; however, he developed severe secondary pancreatitis. Despite appropriate therapy, the patient's leukocyte count and temperature remained elevated, and a pancreatic abscess was suspected. Fourteen days after the onset of symptoms, an In-111-WBC scan demonstrated abnormal uptake in the area of the 
pancreas, which indicated an abscess (Fig. 4). Ultrasonography and abdominal CT scanning showed signs of pancreatitis, with no evidence of abscess. Because of conflicting data, surgery was deferred. The patient's condition gradually improved, and he was discharged. On follow-up CT scanning 5 weeks later, a 4-cm. pseudocyst had developed in the head of the pancreas. Repeat CT scans showed no change in the size of the pseudocyst. Almost 4 months after the onset of symptoms, cystojejunostomy was performed for pseudocyst drainage. There was no evidence of abscess or infection.

\section{False-negative scans}

Two scans were negative for abscesses, but abscesses were present on subsequent surgery. In both patients, signs of infection had been clinically manifested for 2 weeks or more prior to the scans ( 30 days in a patient with a perisplenic abscess and left empyema, and 14 days in a patient with pancreatic abscess). The false-positive scan previously reported and 1 of the false-negative scans both occurred in the same patient.

Case 5. After drainage of a noninfected pancreatic pseudocyst, the 69-year-old male patient discussed in case 4 developed back pain, nausea, fever, and an elevated leukocyte count. Nineteen days after the onset of symptoms, an In-111-WBC scan was negative (Fig. 5A). Because of the discordance between the clinical impression and the scan, a Ga-67 scan was obtained 1 day later; this showed increased activity in the area of the pancreas that was compatible with an abscess (Fig. 5B). Four days later, a pancreatic abscess was found at surgery.

\section{Other findings}

In addition, 9 areas of abnormal uptake that indicated inflammation were seen on 7 scans in 6 patients (Table 1). Areas of inflammation adjacent to abscesses were excluded from this group. One truepositive scan for pelvic abscess also showed inflammation in the buttocks at sites of intramuscular injections. One false-negative scan for perisplenic abscess and empyema showed inflammation in a healing surgical wound. One true-negative scan for an intra-abdominal abscess also demonstrated inflammation in the buttocks.

Case 6. A 41-year-old woman was readmitted 6 days after cholecystectomy with abdominal pain and an elevated leukocyte count. An In-111-WBC scan showed no evidence of abscess; however, there was abnormal uptake in both buttocks corresponding to sites of intramuscular analgesic injections (Fig. 6). Abdominal exploration revealed a bile leak
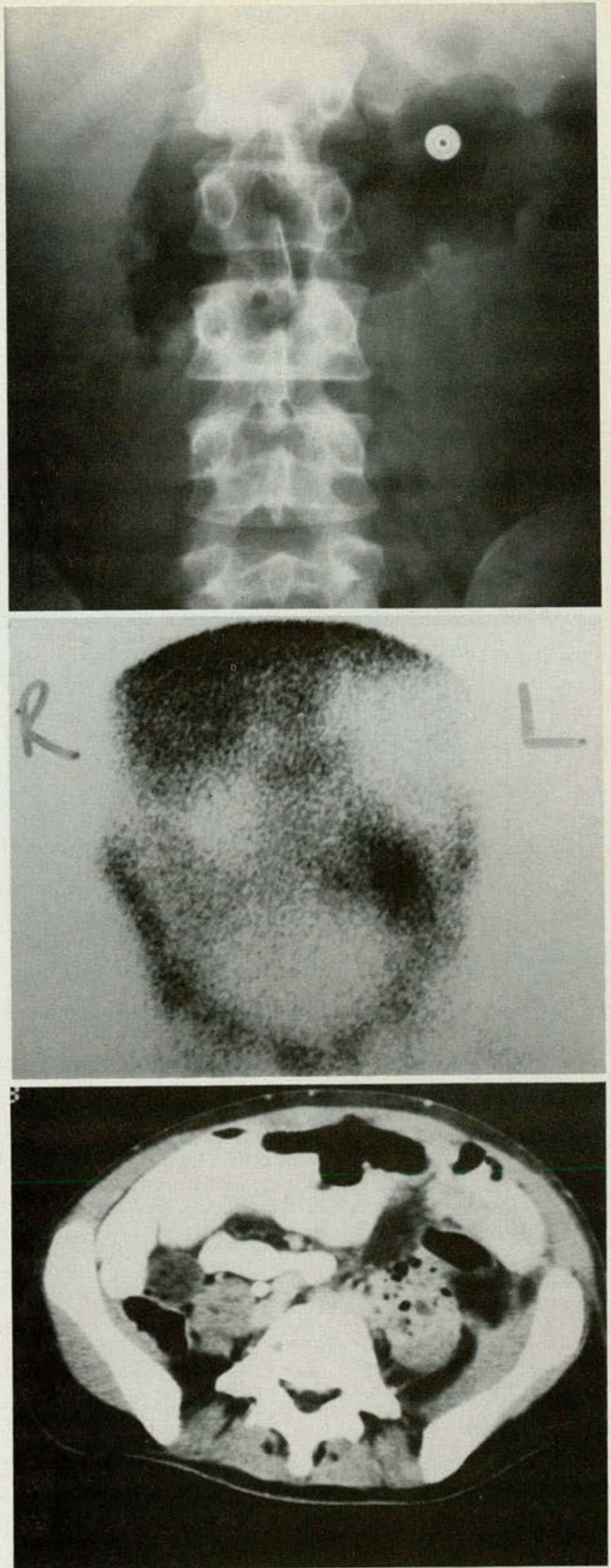

Figs. 2A-2C. Case 2. [A], Plain film of the abdomen shows gas bubbles along the left psoas; these were suspected to be extraluminal. [B], Anterior lower abdominal image of In-111-WBC scan demonstrates abnormal uptake in the left lower quadrant consistent with an abscess. [C], CT scan through the lower abdomen shows enlargement of the left psoas, with gas bubbles present within the muscle. 


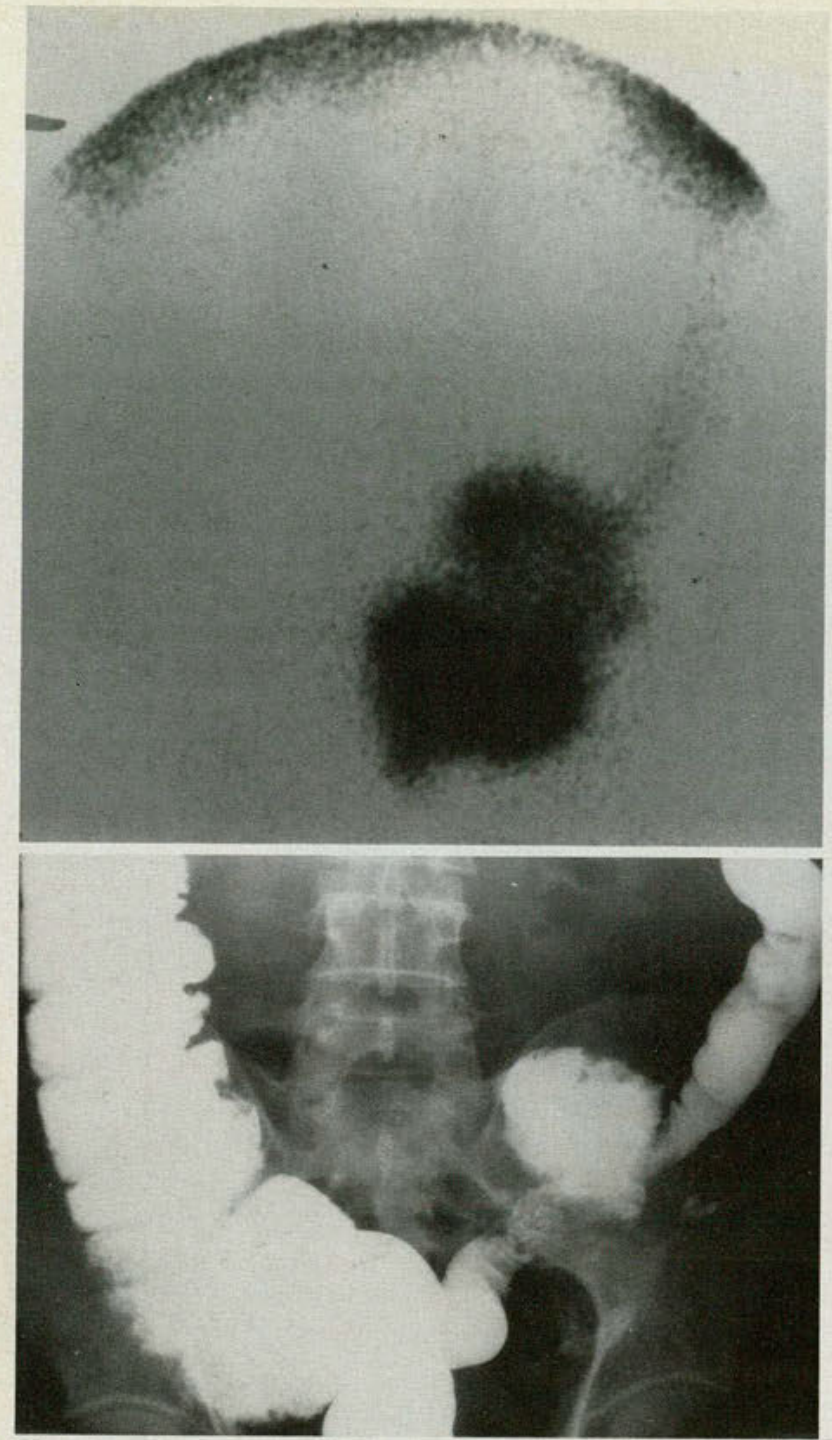

Figs. 3A and 3B. Case 3. [A], Anterior lower abdominal image of an In-111-WBC scan shows a lobulated area of abnormal uptake in the left lower quadrant indicating abscess. Less intense uptake in the descending colon suggests inflammation. [B], Barium enema shows extravasation of contrast into a large cavity, as well as a track of contrast medium that leads to the left anterior abdominal wall.

from the gallbladder fossa, with no evidence of abscess.

\section{Discussion}

Because abscesses are localized collections of leukocytes, it seemed reasonable that leukocytes labeled with isotopes could identify abscesses by their migration. After several unsuccessful attempts by others, ${ }^{10-12}$ McAfee and Thakur ${ }^{13,14}$ found indium-111 oxine to be the most suitable isotope for labeling leukocytes. Successful results were first obtained in animal experiments, ${ }^{15,16}$ then Segal and Thakur and associates ${ }^{1}$ reported the localization of abscesses in humans utilizing In-111-WBCs.

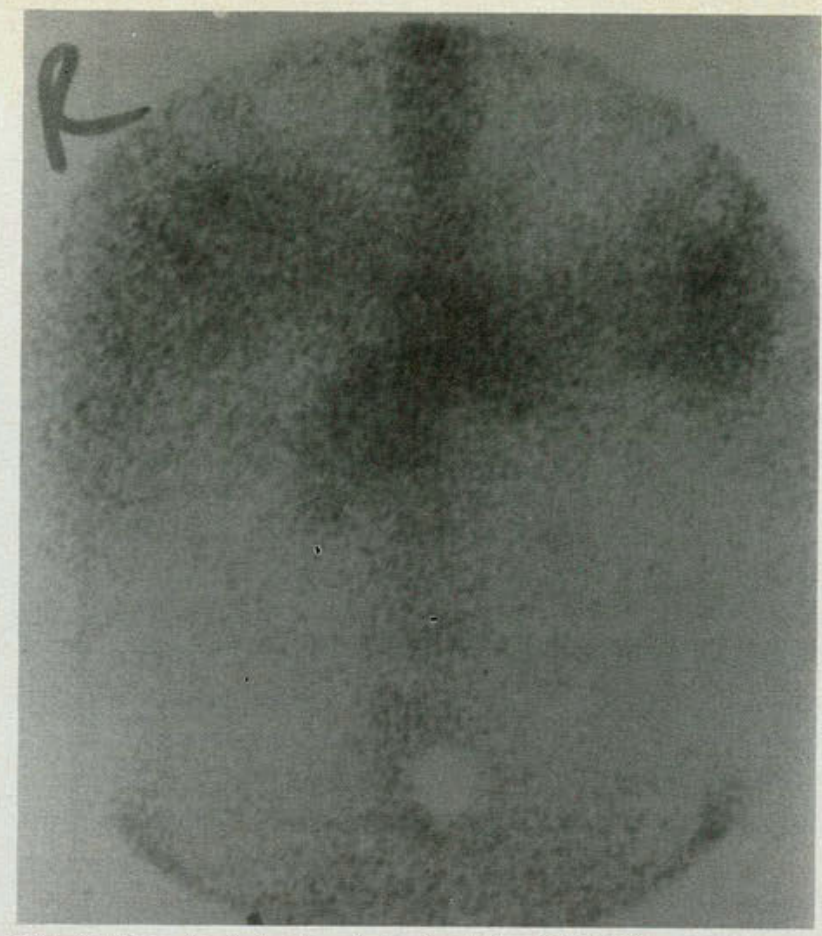

Fig. 4. Case 4. Anterior abdominal In-111-WBC scan demonstrates increased uptake in the area of the pancreas consistent with abscess. Subsequent surgery did not reveal evidence of $a b$ scess or infection.

\begin{tabular}{|c|c|c|}
\hline $\begin{array}{l}\text { Scan } \\
\text { result }\end{array}$ & No. & $\begin{array}{c}\text { Final } \\
\text { diagnosis }\end{array}$ \\
\hline $\begin{array}{l}\text { True-negative } \\
\text { True-positive }\end{array}$ & $\begin{array}{r}16 \\
9\end{array}$ & $\begin{array}{l}\text { Intraperitoneal abscess (6) } \\
\text { Retroperitoneal abscess (2) } \\
\text { Groin abscess (1) }\end{array}$ \\
\hline False-positive & 1 & Pancreatitis \\
\hline False-negative & 2 & $\begin{array}{l}\text { Pancreatic abscess (1) } \\
\text { Perisplenic abscess with empyema (1) }\end{array}$ \\
\hline Other* & 9 & $\begin{array}{l}\text { Buttock inflammation at sites of } \\
\text { intramuscular injection (2) } \\
\text { Lung inflammation in adult } \\
\text { respiratory distress syndrome (2) } \\
\text { Inflammation at exit sites of } \\
\text { gastrostomy tubes (2) } \\
\text { Inflammation at site of healing } \\
\text { surgical wound (1) } \\
\text { Inflammation of cecum and terminal } \\
\text { ileum in Crohn's disease (1) } \\
\text { Thigh inflammation from femoral } \\
\text { vein heroin injection in a } \\
\text { drug addict. (1) }\end{array}$ \\
\hline \multicolumn{3}{|c|}{$\begin{array}{l}\text { *These findings were present on } 7 \text { scans in } 6 \text { patients. Three patients } \\
\text { with inflammation had unrelated findings, which also classified them } \\
\text { as belonging in another group ( } 1 \text { true-positive, } 1 \text { true-negative, } 1 \text { false- } \\
\text { negative). }\end{array}$} \\
\hline
\end{tabular}

Subsequently, these results were confirmed by others. $^{2-8}$

Indium-111 has several qualities that make it suitable for leukocyte imaging. First, it has a halflife of 67 hours, which is long enough to allow for 

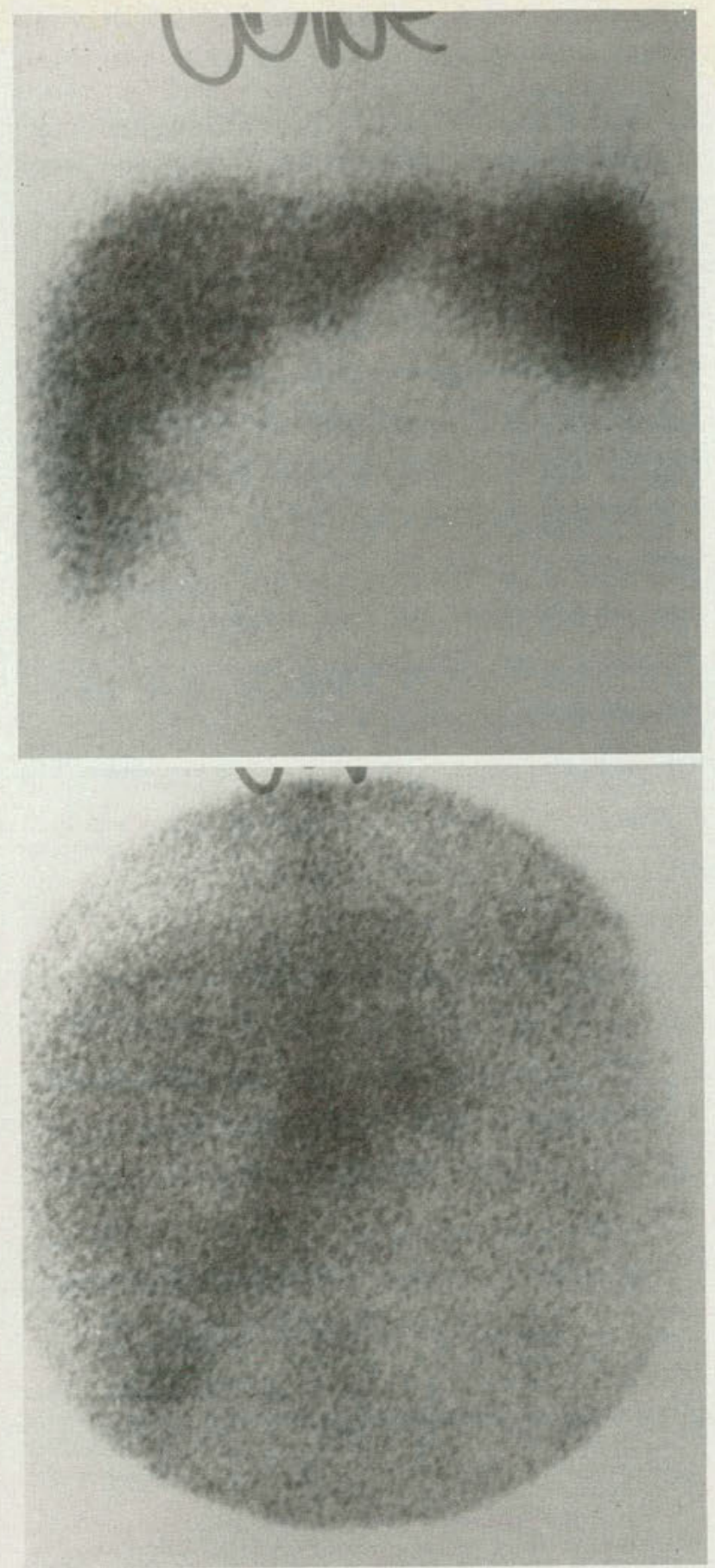

Fig. 5A and 5B. Case 5. [A], In-111-WBC scanning of the same patient reported in case 4 was performed 19 weeks after the initial study. Anterior abdominal image shows no areas of abnormal uptake. [B], Anterior abdominal image of Ga-67 scanning performed the next day reveals abnormal activity in the area of the pancreas.

leukocyte migration to pyogenic processes. Second, indium-111 emits two gamma photons of $173 \mathrm{kev}$ and $247 \mathrm{kev}$, which can be detected with a gamma camera. Third, in the concentrations used, Indium-111 does not alter cell viability or function. ${ }^{17}$
Finally, the radiation dose is acceptable, with the spleen receiving $18 \mathrm{rads} / \mathrm{mCi}$, the liver $1-5$ rads/ $\mathrm{mCi}$, and the whole body $0.5 \mathrm{rads} / \mathrm{mCi} .^{18}$

To cross the leukocyte membrane, indium- 111 must be combined with the chelating agent oxine (8-hydroxyquinoline). The leukocytes must be removed from plasma, because indium-111 has a higher affinity for transferrin than for oxine and dissociates in plasma. In the absence of plasma, indium-111 oxine diffuses across the leukocyte membrane. The indium-111 then dissociates from the oxine and binds to various cytoplasmic components. ${ }^{19}$

In-111-WBC scanning compares favorably to other imaging modalities. In 128 patients studied with In-111-WBCs, Seabold and coworkers ${ }^{20}$ reported a sensitivity of 88 percent, specificity of 90 percent, and accuracy of 89 percent. Carroll and associates $^{6}$ compared In-111-WBC scanning with ultrasonography and documented a sensitivity of 84 percent and a specificity of 95 percent for the In-111-WBC procedure, versus 81 percent sensitivity and 95 percent specificity for ultrasonography. Studying 170 patients with CT, ultrasonography, and In-111-WBC scanning, Knochel and coauthors ${ }^{4}$ reported accuracies of 96 percent, 90 percent, and 92 percent respectively. Sfakianakis and coresearchers ${ }^{8}$ reported a sensitivity of 73 percent and specificity of 98 percent for scanning with In-111-WBCs, as compared to 81 percent sensitivity and 91 percent specificity for scanning with Ga-67.

Ga-67 scanning for abscess detection has several drawbacks. A considerable amount of gallium is excreted through the gut; therefore, caution must be utilized in evaluating abdominal collections of Ga-67. Frequently, 48 to 72 hours are required before interpretation is possible because of high background activity. Ga- 67 also accumulates in certain neoplasms, thus making its uptake equivocal when one is attempting to differentiate a neoplastic from an inflammatory process.

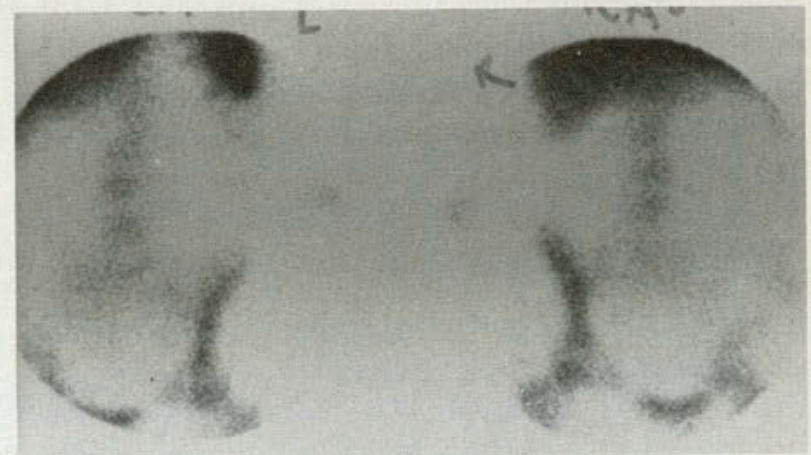

Fig. 6. Case 6. Supine left and right anterior oblique In-111-WBC abdominal scans show faint uptake in both buttocks, indicating inflammation. 
In-111-WBC scanning for abscesses is not without potential pitfalls. Positive scans have been reported in cerebral infarction, adult respiratory distress syndrome, and cystic fibrosis. ${ }^{21}$ Positive scans have also been described in bowel infarction, ${ }^{22}$ acute gastrointestinal hemorrhage, ${ }^{23}$ hematomas, ${ }^{24}$ and in pancreatitis. ${ }^{7}$ In-111-WBC uptake, usually faint, has been described around tracheostomy tubes, at the exit sites of drains or intravascular catheters, and in recent surgical wounds. ${ }^{25}$ Although In-111-WBC uptake in neoplasm is rare, it has been reported. ${ }^{2,20,25-27}$ The above list of false-positives scans is not complete. As well as reviewing the literature, McAfee and Samin $^{25}$ compiled their own list of false-positives.

False-negative scans also occur with In-111-WBC scanning. Granulocytopenic patients have a potential for false-negative studies, and McDougall, Baumert, and Lantieri ${ }^{2}$ recommended that patients with polymorphonuclear leukocyte counts $<$ $2,000 / \mathrm{cu}$. mm. should probably be studied with labeled donor cells. Perhaps the greatest potential for false-negative scans occurs in abscesses of long duration. For abscesses more than 2 weeks old, In-111WBC scintigraphy may be falsely negative, 8,28 and in one study, ${ }^{8} 7 / 26$ (27 percent) of false-negative In-111-WBC scans occurred in septic foci of greater than 2 weeks' duration.

In the present series, 32 In-111-WBC scans from 28 patients with suspected abscesses were reviewed. In-111-WBC sensitivity was 82 percent, specificity 94 percent, and accuracy 89 percent. There was 1 false-positive scan in a patient with fulminant pancreatitis. This occurrence has been previously reported. ${ }^{7}$ Anderson and associates ${ }^{29}$ reported that positive scans are seen only in severe, not in mild, pancreatitis. There were two falsenegative scans. In both cases, septic foci had been manifested clinically for longer than 2 weeks prior to scanning, which is consistent with the reports that infections older than 2 weeks may produce scans that are falsely negative.

Twenty-two percent (7/32) of all scans showed areas of inflammation that were unrelated to abscesses. In-111-WBC uptake in inflammation is less intense than the liver uptake, and areas of abnormal uptake must be compared to the liver uptake to avoid false-positive interpretation.

\section{Summary}

Indium-111-leukocyte scintigraphy is an accurate, noninvasive test for the detection of abscesses, especially acute processes. The whole body can be imaged when searching for occult infections. In-111-WBC scans are easier to interpret than Ga-67 scans because of the lack of uptake by the kidney or bowel. Also, diagnostic In-111-WBC scans can be obtained sooner after injection than Ga-67 scans. False-positives and false-negatives results do occur. Although In-111-WBC scanning can detect chronic abscesses, the sensitivity decreases when the abscess is older than 2 weeks. 1. Segal, A.W., et al.: Indium-111-labelled leucocytes for localisation of
abscesses. Lancet $2: 1056-8,13$ Nov 76

2. McDougall, I.R., Baumert, J.E., and Lantieri, R.L.: Evaluation of ${ }^{{ }^{1} \text { In }}$ leukocyte whole body scanning. AJR 133:849-54, Nov 79

3. Coleman, R.E., et al.: Indium-111-labeled leukocytes in the evaluation of suspected abdominal abscesses. Am J Surg 139:99-104, Jan 80

4. Knochel, J.Q., et al: Diagnosis of abdominal abscess with computed tomography, ultrasound, and "111n leukocyte scans. Radiology 137:425-32, Nov 80

5. Dutcher, J.P., Schiffer, C.A., and Johnston, G.S.: Rapid migration of 111indium-labeled granulocytes to sites of infection. $\mathrm{N}$ Engl J Med 304:586-9, 5 Mar 81

6. Carroll, B., et al:: Ultrasonography and indium-111 white blood cell scanning for the detection of intraabdominal abscesses. Radiology 140:155-60, Jul 81

7. Bicknell, T.A., Kohatsu, S., and Goodwin, D.A.: Use of indium-111labeled autologous leukocytes in differentiating pancreatic abscess from pseudocyst. Am J Surg 142:312-6, Sep 81

8. Sfakianakis, G.N., et al.: Comparisons of scintigraphy with In-111 leukocytes and Ga-67 in the diagnosis of occult sepsis. J Nucl Med 23:618-26, Jul 82

9. Beightol, R.W., and Baker, W.J.: Labeling autologous leukocytes with indium-111 oxine. Am J Hosp Pharm 37:847-50, Jun 80

10. Allen, H.M., Bergner, R., and Dusault, L.A.: Localization of deep abscesses with radioactive isotopes. Henry Ford Hosp Med J 21:111-6, 1973 11. English, D., and Anderson, B.R.: Labeling of phagocytes from human blood with ${ }^{99 m}$ Tc sulfur colloid. J Nucl Med 16:5-10, Jan 75

12. Burleson, R.L., Holman, B.L., and Tow, D.E.: Scintigraphic demonstration of abscesses with radioactive gallium labeled leukocytes. Surg Gynecol Obstet 141:379-82, Sep 75

13. McAfee, J.G., and Thakur, M.L.: Survey of radioactive agents for in vitro labeling of phagocytic leukocytes. 1. Soluble agents. J Nucl Med 17:480-7, Jun 76

14. McAfee, J.G., and Thakur, M.L.: Survey of radioactive agents for in vitro labeling of phagocytic leukocytes. II. Particles. J Nucl Med 17:488-92, Jun 76

15. Thakur, M.L., Coleman, R.E., and Welch, M.J.: Indium-111-labeled leukocytes for the localization of abscesses. Preparation, analysis, tissue distribution, and comparison with gallium-67 citrate in dogs. J Lab Clin Med 89:217-28, Jan 77

16. Thakur, M.L., et al.: Preparation and evaluation of ${ }^{111}$ In-labeled leukocytes as an absess imaging agent in dogs. Radiology 119:731-2, Jun 76 17. Zakhireh, B., et al.: Indium-111-labeled human polymorphonuclear leukocytes. Viability, random migration, chemotaxis, bactericidal capacity and ultrastructure. J Nucl Med 20:741-7, Jul 79

18. Coleman, R.E. Radioactive leukocytes. In Nuclear medicine annual 1982, edited by L.M. Freeman and H.S. Weissman, Raven Press, New York, 1982, pp. 119-41

19. Thakur, M.L., et al.: Indium-111-labeled cellular blood components. Mechanism of labeling and intracellular location in human neutrophils. J Nucl Med 18:1022-6, Oct 77

20. Seabold, J.E., et al.: Unsuspected extra-abdominal sites of infection. Scintigraphic detection with indium-111-labeled leukocytes. Radiology 151:213-7, Apr 84

21. Coleman, R.E., and Welch, D.: Possible pitfalls with clinical imaging of indium-111 leukocytes. Concise communications. J Nucl Med 21:122-5, Feb 80

22. Gray, H.W., Cuthbert, I., and Richards, J.R.: Clinical imaging with indium-111 leukocytes. Uptake in bowel infarction. J Nucl Med 22:701-2, Aug 81

23. Fisher, M.F., and Rudd, T.G.: In-111-labeled leukocyte imaging. Falsepositive study due to acute gastrointestinal bleeding. J Nucl Med 803-4, Sep 83

24. Wing, V.W., et al.: Indium-111-labeled leukocyte localization in hematomas. A pitfall in abscess detection. Radiology 152:173-6, Jul 84 
25. McAfee, J.G., and Samin, A.: In-111-labled leukocytes. A review of problems in image interpretation. Radiology 155:221-9, Apr 85

26. Schell-Frederick, E., et al.: "11Indium-oxine-labeled leukocytes in the diagnosis of localized infections in patients with neoplastic disease. Cancer 54:817-24, Sep 84

27. Sfakianakis, G.N., et al.: Positive indium-111 leukocyte scintigraphy in skeletal metastasis. AJR 139:601-3, Sep 82

28. Ascher, N.L., et al.: Indium 111 autologous tagged leukocytes in the diagnosis of intraperitoneal sepsis. Arch Surg 114:386-92, Apr 79

29. Anderson, J.R., et al.: Initial experience with indium-111 autologous leukocyte imaging in patients with acute pancreatitis. Br Med J 287:637-8, Sep 83

Accepted for publication in July 1986. Updating, as necessary, has been done by the author.
At the time this paper was written, Dr. Tryciecky was a radiology resident at Botsford General Hospital in Farmington Hills, Michigan; Leo Sheiner, D.O., is the chairman of the Department of Radiology and Nuclear Medicine. Stephan R. Morse, D.O., is the resident trainer. Dr. Tryciecky is now a staff radiologist at the Memorial Hospital in Owosso, Michigan. He is board certified in diagnostic radiology.

Dr. Tryciecky, the Memorial Hospital, 826 W. King Street, Owosso, Michigan 48867. 